DIE FRAU IN DER OPER 


\title{
CATHERINE CLÉMENT
}

\section{DIE FRAU IN DER OPER}

\section{Besiegt, verraten und verkauft}

\author{
Aus dem Französischen \\ von \\ Annette Holoch \\ Mit einem Vorwort \\ von \\ Silke Leopold
}

\author{
J. B. METZLERSCHE \\ VERLAGSBUCHHANDLUNG \\ STUTTGART
}


Die Originalausgabe erschien unter dem Titel

Catherine Clément "L'opéra ou la défaite des femmes «

bei Editions Grasset \& Fasquelle, Paris, 1979.

(C) 1979 Editions Grasset \& Fasquelle, Paris

Die Deutsche Bibliothek - CIP-Einheitsaufnahme

Clément, Catherine:

Die Frau in der Oper: besiegt, verraten und verkauft/

Catherine Clément. Aus dem Franz. von Annette Holoch. Mit

einem Vorw. von Silke Leopold. - Stuttgart: Metzler, 1992

Einheitssacht.: L'opéra ou la défaite des femmes «dt.)

ISBN 978-3-476-00785-8

ISBN 978-3-476-03382-6 (eBook)

DOI 10.1007/978-3-476-03382-6

Dieses Werk einschließlich aller seiner Teile ist urheberrechtlich geschützt. Jede Verwertung außerhalb der engen Grenzen des Urheberrechtsgesetzes ist ohne Zustimmung des Verlages unzulässig und strafbar. Das gilt insbesondere für Vervielfältigungen, Übersetzungen, Mikroverfilmungen und die Einspeicherung und Verarbeitung in elektronischen Systemen.

(C) 1992 Springer-Verlag GmbH Deutschland Ursprünglich erschienen bei J.B. Metzlersche Verlagsbuchhandlung und Carl Ernst Poeschel Verlag GmbH in Stuttgart 1992 


\author{
Für Claude Lévi-Strauss
}

und meinen Sohn Michel 


\section{INHALT}

Vorwort von Silke Leopold Seite 9

Vorspiel Seite 15

1. KAPITEL

Sängerinnen oder der Frauenzirkus Seite 39

2. KAPITEL

Tote Seite 63

3. KAPITEL

Familiengeschäfte oder die schrecklichen Eltern Seite 85

4. KAPITEL

Junge Mädchen und der Sprung ins Leere Seite 107

5. KAPITEL

Furien und Götter oder der abnehmende Mond Seite 131

6. KAPITEL

Wahnsinnige, Neger, Narren oder

die Helden der Enttäuschung Seite 159

7. KAPITEL

Tetralogien oder die verlorene Tochter Seite 183

Lobrede auf das Heidentum Seite 225

Register Seite 235 


\section{VORWORT}

\section{Von Frauen, Riten und Mythen}

Ist die Oper misogyn? Ja, sagt Catherine Clément. Geschaffen von Männern, von Librettisten, Komponisten und Regisseuren, hat sie die Vernichtung der Frauen zum Thema, von Frauen, die dafür büßen müssen, daß sie die heile (Gefühls)welt der Männer durcheinanderbringen - besiegt wie die Königin der Nacht, verraten wie Madame Butterfly und verkauft wie Violetta Valéry. Gemordete und dem Wahnsinn verfallene, verführte und verlassene, vergiftete und mißhandelte Frauen: Das ist der Stoff, aus dem Opern sind.

Ist die Oper misogyn? Nein, sagt Catherine Clément. Denn die Oper rührt an Schichten der menschlichen Erfahrung, die dem Kopf verschlossen bleiben, sie weist den Frauen Wege aus der Gefangenschaft des (männlichen) Intellekts. Indem sie die Hysterie auf der Bühne vorführt, erlaubt sie den Frauen im Parkett, in gleicher Weise darauf zu reagieren, ihre eigene, spezifisch weibliche Welterfahrung an den Schicksalen jener Frauen zu messen, denen ihre Stärke oder ihre Schwäche zum Verhängnis wird. Die Oper, sagt Catherine Clément, kommt aus der Gebärmutter.

Aus diesem scheinbaren Widerspruch heraus ist ein Buch entstanden, das keinen Leser gleichgültig lassen wird, das Zustimmung und Ablehnung hervorrufen wird, vielleicht auch - ich bekenne es - beides gleichzeitig; in jedem Falle aber ein Buch zum Weiterdenken, mit Bedacht tendenziös in der Auswahl des Materials, bewußt subjektiv in der Deutung. Oper - das ist für die Autorin das Geschehen auf der Bühne ebenso wie die Reaktionen des Publikums, die Sänger(innen) ebenso wie die Rollen, die sie verkörpern - ein Ritual, das sich allabendlich wiederholt; ein Ritual, bei dem die Frauen unten im Parkett keine Stimme haben, sondern nur schmückendes Beiwerk sind, während die Frauen auf der Bühne den Saal mit ihrer Stimme füllen, um ihre eigene Niederlage zu besingen. Als geknechtete, von Männern ins Verderben getriebene Sängerinnen passieren da die Malibran und die Callas, Antonia und Tosca Revue; gleichermaßen dem Tode geweiht erscheinen so unterschiedliche Frauentypen wie Madame Butterfly und Carmen, Isolde und Mélisande. Ein Opfer familiärer Konstellationen ist Elisabeth von Valois ebenso wie Violetta Valéry, Pamina und Elektra, und von den Wirrnissen der Mädchenseele erzählen Tatjana und Mimi, Lucia di Lammermoor und Elvira, die Puritanerin. Turandot und Norma, die Marschallin und Amneris, Brünnhilde und Kundry stehen für die besiegten Frauen, deren Leben seinen Sinn durch einen Mann bekommt und verliert. Auch ein paar Männer kommen in dem Buch vor; freilich sind es Männer, denen, so Clément, Frauengeschichten wi- 
derfahren - Männer, die dem Verderben geweiht sind, ausgeschlossene, verletzte Männer: Otello, Falstaff und Hans Sachs.

Was aber macht uns so neugierig auf die Wechselfälle einer schwindsüchtigen Näherin, einer alternden Druidenpriesterin - auf Themen, die mit unserer Wirklichkeit scheinbar so wenig zu tun haben, die auf der Theaterbühne oder im Roman kaum mehr ein Publikum finden würden? Es ist die Musik, die uns alle diese Schicksale nahebringt, die uns verrät, was die Worte bisweilen zu verbergen suchen; die Musik, aus der heraus die Texte erst ihren Sinn bekommen, die den Texten über das Einzelschicksal hinaus allgemeine Gültigkeit verleiht, die den Schicksalsfaden zwischen der Bühne und dem Publikum spinnt. Kein Wunder, daß der Musik in der Oper bisher größere Aufmerksamkeit zuteil wird als den Texten. Clément freilich nimmt zuallererst die Libretti ins Visier, denen sie ähnliche Qualitäten attestiert wie der Musik - das Bewußtmachen des Unbewußten, der Grundmuster menschlichen Miteinanders. Sie interpretiert die Libretti in strikt feministischer Weise und kommt dabei zu verblüffend originellen Einsichten in altbekannte Stoffe. Wer würde schon darauf kommen, daß der Schlüssel zu Tschaikowskys Eugen Onegin in der Marmelade zu suchen ist? Clément aber entwirft mit faszinierender Stringenz ein Bild von der Marmelade, um deren Bereitung es im ersten Bild der Oper geht, als Metapher für den Verzicht auf Leidenschaft, für die Resignation der jungen, schwärmerischen Tatjana in einer bürgerlichen Existenz. Oder wer würde in der Chromatik von Isoldes Liebestod ein Bild des Regenbogens vermuten, der Isolde als Spenderin des Guten wie des Bösen ausweist?

Es ist ein sehr französisches Buch, dieser Opernführer durch die weibliche Brille, das nun in deutscher Übersetzung sein Publikum sucht; französisch auf mehrerlei Weise: zum einen in der Darstellung, die Wissenschaftliches und Literarisches, Erkenntnisse und Assoziationen in einer Weise vermengt, die dazwischen zu unterscheiden kaum mehr erlaubt. Einem deutschen Leser wird es möglicherweise zunächst schwerfallen, die Spreu der Information vom Weizen der Interpretation zu trennen; es mag ihm seltsam erscheinen, die Königin der Nacht und Sarastro als Ehepaar wiederzufinden; der vermeintliche Irrtum entschleiert sich jedoch im Laufe von Cléments Ausführungen als eine bewußte Fiktion, als das Bild, das Clément hinter der Geschichte, wie sie auf der Bühne passiert, zu sehen vermeint - die Geschichte einer Familie, die sich um eine Tochter streitet. Wer sich auf dieses gänzlich andere Denken, auf diese gänzlich andere, spezifisch französische Zugehensweise einläßt, wird den Wert dieses Buches erkennen und in der Auseinandersetzung mit seinen Thesen, auch wenn er die Prämissen nicht nachvollziehen kann, doch von den Ergebnissen und für die eigenen Denkmuster profitieren. 
Französisch ist Cléments Buch auch in seinem philosophischen Ansatz. Denn die feministische These ist nur die eine Schicht ihrer Darstellung; sie wird überlagert und durchdrungen von dem Versuch, die Erkenntnisse und Methoden des Strukturalismus und der Mythenforschung ihres Lehrers Claude Lévi-Strauss auf die Oper anzuwenden; Isoldes Chromatik und der Mythos von der Entstehung des Regenbogens bei den Amazonas-Indianern würden demnach auf ein gemeinsames Grundmuster verweisen. Nicht von ungefähr bildet Wagners Ring-Tetralogie, die Lévi-Strauss selbst als eine erste Strukturanalyse der Mythen bezeichnete, den Höhepunkt ihrer Darstellung: An Wagners Mythen, Erzählungen des Unbewußten, wo Text und Musik, Inhalt und Sprache, Handlungs- und musikalische Motive in vielfältiger Weise aufeinander bezogen und gegeneinander verschoben sind, kann Clément die altbekannte Erkenntnis, daß die Oper von Grundkonstellationen des menschlichen Miteinanders handelt, daß die Musik zwischen den Zeilen des Textes zu lesen vermag, daß sie den Text unterstützen, aber auch konterkarieren kann, daß sie eine unmerkliche, aber unmittelbare Verbindung zu der Wahrnehmungswelt des Zuschauers schafft, in einem neuen Licht beschreiben: im Licht jener kulturellen Analogien, jener elementaren Strukturen, die jedes Individuum, jede Gesellschaft über alle historischen und geographischen Grenzen hinweg mit allen anderen verbindet.

Gehört die Vernichtung der Frau dazu? Ja, sagt Catherine Clément. Jedenfalls der Frauen, die sich außerhalb der Gesetze der Familien und Strukturen stellen: die Zigeunerin, die Prostituierte, die Polizistenmörderin. Die Welt der Männer duldet keine Unordnung. Ist aber die Ordnung wiederhergestellt, wenn Carmen, Violetta, Tosca ihr Leben gelassen haben? Und wieviel Männer müssen in der Oper ihr Leben lassen, weil sie sich außerhalb der Regeln stellen? Was unterscheidet das Schicksal Turriddus aus der Cavalleria rusticana von dem der Nedda aus dem Bajazzo? Und ist Riccardo aus dem Ballo in maschera weniger ein Opfer als Maria Stuarda bei Donizetti? Was ist schließlich mit den starken Frauen, der Leonore aus Beethovens Fidelio, und was mit den Paaren, die gemeinsam für verbotene Liebe büßen müssen?

Wer auf solche Fragen Antworten sucht, wird von Cléments Buch enttäuscht sein. Erwarten Sie keinen Überblick über die Operngeschichte, über die Frau in der Oper von ihren Anfängen bis heute; Cléments Auswahl ist nicht nur tendenziös, sie ist auch eng. Susanna aus Mozarts Figaro, Leonore aus Beethovens Fidelio, Agathe aus Webers Freischütz - sie alle hätten keinen Platz im Kreis der besiegten Frauen. Auf die Oper des 18. Jahrhunderts paßt ihre These ebensowenig wie auf viele Werke der zeitgenössischen Musik. Die Opera buffa mit ihren starken, listigen Frauen muß gänzlich ausgespart bleiben wie auch die Opera seria, die generell kaum Besiegte kennt, es sei denn, jemand besiege 
sich selbst. Das Repertoire dieses Buches beschränkt sich, von wenigen Ausnahmen abgesehen, auf die Opern des 19. und beginnenden 20. Jahrhunderts. In ihrem strukturalistischen Ansatz leugnet Clément die Historizität der Oper, ihre Wandlungen, ihre Vielfalt, ihre Widersprüchlichkeit; und die Spielpläne der Opernhäuser von heute, die genau dieses Repertoire bevorzugen, geben ihr weltweit recht.

$\mathrm{Da}$ gerade in diesem Repertoire auffallend viele Frauen ihr Leben lassen müssen, läßt sich nicht leugnen; mit sicherer Hand wählten die Opernmacher aus dem reichen Schatz der möglichen Sujets, aus der antiken Mythologie, der Historie oder der Literatur gerade solche Stoffe aus, in denen Frauen zu Tode kommen - aber auch Männer. Welchen Anteil hat daran die bürgerliche Gesellschaft? Ist es nicht auch der Sittenkodex, der eine starke Leidenschaft, ein Außer-Sich-Geraten, ein Außer-Kraft-Setzen der Regeln des menschlichen Miteinanders nicht mehr duldet? Der Bestrafung verlangt statt, wie im 18., dem Jahrhundert des Happy ending, Vergebung? Und sind es nicht gerade jene Leidenschaften, die überquellenden Herzen, die die Oper überhaupt erst möglich machen? Was rechtfertigte den Gesang, wenn nicht die Aufwallung der Gefühle? Die Oper handelt per definitionem vom Überschreiten der gesellschaftlichen Regeln, von Extremsituationen jenseits der Contenance.

Sicher, wer den eigenen Passionen freien Lauf läßt, muß dafür büßen. Das verlangt die Gesellschaft, die sonst in ihrer Ordnung, in ihrem Fortbestand gefährdet wäre. Doch sind es nicht gerade die Komponisten, die Partei nehmen für jene, denen das Herz übergeht? Spricht nicht gerade aus der Musik so etwas wie eine leise Sehnsucht, ein wenig Neid auf die Fähigkeit der Opfer, ihre Leidenschaften bis zum Exzeß, dem die Vernichtung folgt, auszuleben? Gewiß entzündete sich die musikalische Phantasie eines Komponisten eher an solchen Exzessen als an der matten Funzel des Anstandes. Auch Titus, dieses Muster an Selbstbeherrschung, singt erst in Momenten, wenn ihm diese verlorenzugehen droht.

Daß es nur den Frauen gestattet war, außer sich zu geraten, war freilich nicht immer so. Die Opera seria gedachte dem jeweils Edelsten den größten Raum für Emotionen zu - unabhängig davon, ob diese Hauptperson der Oper ein Mann oder eine Frau war. Es war die Zeit, in der Männer sich schminkten und puderten, in der sie Stärke zeigten, wenn sie weinten. In Händels Opern steht einer Alcina noch ein Bajazet gegenüber - zwei Opfern, die vormals Täter waren, deren Verzweiflungs- und Wutausbrüche sich in nichts nachstehen. Schon bei Mozart, wenn nicht gar bereits in Händels Oratorien, wird ein Wandel spürbar. Mozarts Frauengestalten ist eine weit größere Bandbreite der Gefühle, der Tonarten und Tempi eigen als seinen Männergestalten; an die Rase- 
reien einer Elettra, einer Vitellia oder gar einer Dorabella reicht so leicht kein Mann in Mozarts Opern heran. Erst im 19. Jahrhundert mit seinen neuen Idealen von Männlichkeit und Weiblichkeit wurde daraus eine Methode; nun riskierte sein Leben, wer die Ordnung, die gesellschaftliche wie die emotionale, störte; denn nun war jeder selbst für das Funktionieren des Gemeinwesens verantwortlich, gab es doch keinen Herrscher mehr, dessen Vergebung eine ausweglose Situation, eine Schuld hätte aufheben können. Und nun gingen die Frauen voran, wenn es ans Sterben ging. Doch die Leidenschaften, das Herausschreien von Angst und Schmerz, wie es Clément für die Frauen in der Oper in Anspruch nimmt, verteilten sich bei näherer Betrachtung eher gleichmäßig auf Frauen und Männer - auch auf Manrico, den Troubadour, der die Geliebte und Mutter die verliert, auf Rigoletto, der für den Tod seiner Tochter mit dem Weiterleben bestraft wird, auf Eléazar aus Halévys Jüdin, der die Tochter dem Glauben opfert. Sie alle als Männer zu vereinnahmen, die Frauenschicksale erleiden, hieße eine an sich faszinierende These überstrapazieren.

Die Qualität dieses Buches bemißt sich freilich nicht nach dem, was es ausläßt, sondern nach dem, was es tatsächlich enthält. Catherine Clément lädt uns ein, bekannte, bisweilen allzu bekannte Opern aus einem neuen Blickwinkel $\mathrm{zu}$ betrachten, Personenkonstellationen neu zu sortieren und uns selbst, unsere eigenen Wurzeln dabei im Spiegel der Opern-Mythen zu erkennen. Ihr Buch, als »Übergangsritus« für ihren Sohn geschrieben, kann auch für uns, die Leser, einen Schritt in eine neue Erkenntniswelt bedeuten. 